\title{
Microbiological assay for quantitative determination of Imipenem in powder for injection
}

\author{
Sendy Sales Oliveira ${ }^{\mathrm{a}}$, Fábio de Souza Barbosa ${ }^{\mathrm{b}^{*}}$, Leonardo Capra Pezzi ${ }^{\mathrm{a}}$, \\ Elfrides E. S. Schapoval ${ }^{\mathrm{a}, \mathrm{b}}$, Andreas S. L. Mendez ${ }^{\mathrm{a}, \mathrm{b}}$
${ }^{a}$ Faculdade de Farmácia, Universidade Federal do Rio Grande do Sul, Av. Ipiranga 2752, CEP 90610-000, Porto Alegre - RS, Brasil 90610-000, Porto Alegre - RS, Brasil \\ ${ }^{b}$ Programa de Pós-graduação em Ciências Farmacêuticas, Universidade Federal do Rio Grande do Sul, Av. Ipiranga 2752, CEP \\ *Corresponding author: fabiodsbarbosa@yahoo.com
}

This work describes the development and validation of a microbiological method using the cylinder-plate assay for quantitative determination of imipenem in powder for injection. The aim was to obtain a low-cost and suitable methodology that can be alternative to physicochemical techniques already described, contributing for the quality control of this antibiotic. Firstly, the analytical conditions were optimized, testing the microorganism, inoculum concentration and best range of sample and standard concentrations, in a way that provides the adequate measurement of the inhibition halos. Staphylococcus epidermidis ATCC 12228 was selected as test microorganism, as well as $2.0 \%$ of inoculum concentration. The validation protocol followed the official guidelines, and the parameters evaluated were linearity, precision (intermediate precision and repeatability) and accuracy. All standard curves ranging $0.5-2.0 \mu \mathrm{g} / \mathrm{mL}$ showed $\mathrm{r}$ values higher than 0.999 , and ANOVA confirmed that were no deviation from linearity ( $p$-value $>0.05$ ). The method also proved to be precise with RSD (relative standard deviation) values ranging $0.28-0.64$ for repeatability and 2.49 for intermediate precision. It was performed three days of experiments, being three assays of eight plates a day. The drug mean content was $101.05 \%$. Accuracy was assessed by recovery test, with standard recovery percentage of $101.70-107.90 \%$ (mean recovery $=104.86 \%$ ), which was considered satisfactory. Therefore, the proposed microbiological method was considered validated and suitable for application in quantitative determination of this drug, being useful for quality control routine.

Keywords: imipenem, microbiological assay, quantitative determination, validation, quality control.

\section{Introduction}

Imipenem (Figure 1), or chemically $(5 \mathrm{R}, 6 \mathrm{~S})-3-[2-$ (aminomethylideneamino) ethylsulfanyl]-6-[(1R)-1hydroxyethyl]-7-oxo-1-azabicyclo [3.2.0] hept-2-ene-2carboxylic acid, is a broad spectrum beta-lactam antibiotic belonging to the subgroup of carbapenems, which are drugs structurally composed of a pentacyclic thiazolidinic ring attached to the nitrogen of the beta-lactam ring by a tetrahedral carbon atom (1). Derived from thienamycin, imipenem is the oldest carbapenems available for clinical use, approved in 1986 in the USA (2). It has activity against most of the Gram-positive and Gram-negative, aerobic and anaerobic bacteria (1), including those beta-lactamaseproducing bacterial pathogens. This occurs due the greater stability of pentacyclic ring that is attached to beta-lactam ring against the action of these enzymes (3). However, drugresistance has been reported in Gram-negative bacteria, for example in some strains of Pseudomonas aeruginosa (4). Clinically, imipenem is used to treat several polymicrobial infections, such as pneumonia and complicated gastrointestinal tract infections. The recommended dose is 2 $\mathrm{g}$ /day in adults with normal renal functions (4). However, imipenem is hydrolyzed by a renal dehydropeptidase (dehydropeptidase I) (3), being inactivated, requiring coadministration of cilastatin, an inhibitor of this enzyme in clinical use. Imipenem and cilastatin are marketed as powder for injection $(1: 1, \mathrm{w} / \mathrm{w})$ in doses of 250 and $500 \mathrm{mg}$, since they are not absorbed orally. Most often they are given intravenously by infusion, but in case of treatment of less severe infections, they can be given intramuscularly (in dose of $750 \mathrm{mg}$ ) (5).

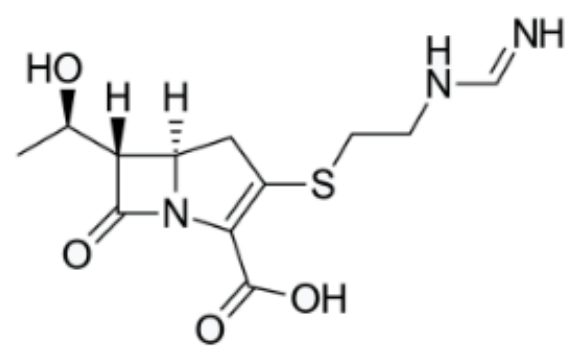

Figure 1. Chemical structure of imipenem.

A review of literature shows many analytical studies applied to imipenem in biological fluids, involving HPLC-DAD (68 ), and spectrophotometric/chemometric technique (9). On the other hand, for pharmaceutical dosage forms, the literature reports derivate spectrophotometric $(10,11)$, and RP-UPLC method for quantitation of imipenem related impurities (12). In fact, antibiotics play an increasingly important role in pharmacotherapy because of their wide use in the clinical practice, so it is extremely important to develop alternative methods that allow their analytical determination in existing pharmaceutical forms (13). 
Microbiological assays have been widely used to determinate antibiotic drugs along the years (13-15). These methods are recommended by several pharmacopoeias, like the USP and European Pharmacopoeias (16-18). Different techniques are described in microbiological assays that are used for antibiotic drugs to determine antibacterial activity, like paper disk procedures, standard broth microdilution assay, and cylinder-plate assay. The evaluation of the inhibitory effect of the antibiotic on the growth of the test microorganisms makes possible its quantitative determination by means of logarithmic relation between the drug concentration and antimicrobial potency (13).

Therefore, microbiological assays can be an alternative to the physicochemical methods like HPLC, not requiring sophisticated equipment or toxic solvents, and being viable to use even in small laboratories. For carbapenems, two microbiological cylinder-plate assays are described for meropenem and doripenem, both developed to determine the drugs in powder for injections $(13,19)$. For imipenem, this methodology is absent. Thus, the present manuscript reports the development and validation of microbiological assay for quantitation of imipenem in powder for injection, applying the cylinder-plate method.

\section{Experimental}

\section{Chemicals}

Imipenem reference standard $(85.5 \%)$ was obtained from Sigma-Aldrich (St. Louis, Missouri, EUA). Powder for injection containing $500 \mathrm{mg}$ of imipenem monohydrate and $500 \mathrm{mg}$ of Cilastatin sodium was purchased from ABL Antibiotics (Cosmópolis, SP, Brazil). For microbiological assay, DifcoTm Antibiotic Media number 11 obtained from Interlab ${ }^{\circledR}$ (São Paulo, SP, Brazil), and Grove Randall number 1 agar from Merck (Darmstadt, Germany) were used. All solutions were prepared in distilled water and sterilized before each experiment. Analytical grade sodium chloride of the Synth LTDA (São Paulo, SP, Brazil) was used for preparation of inoculum suspension.

\section{Microbiological assay}

\section{Microorganism and inoculum}

Staphylococcus epidermidis ATCC 12228 was chosen as microorganism test. The strains were cultivated with number 1 agar and kept in freezer. One day before the assay, the microorganism was inoculated with the same agar that was kept at $35^{\circ} \mathrm{C}$ for $24 \mathrm{hrs}$. The inoculum suspension was prepared using $\mathrm{NaCl} 0.9 \%$ sterile solution, which was standardized with $25 \pm 2 \%$ of transmittance at $580 \mathrm{~nm}$, using a spectrophotometer Analyser ${ }^{\circledR}$ Model 800 (São Paulo, SP, Brazil). $\mathrm{NaCl} 0.9 \%$ solution was used as blank of absorption. Portions of this inoculum suspension were inoculated into erlenmeyer flasks containing number 11 agar in order to obtain a concentration of $2 \%$ inoculum, being kept at $47 \pm 2^{\circ}$ C. Then, $6 \mathrm{~mL}$ of this inoculum $(2 \%)$ were applied over the base layer (composed by $20 \mathrm{~mL}$ of number 11 agar) in the plates.

Standard and sample solutions
Imipenem standard solution was prepared for each experiment. Sterile distilled water was used as solvent for drug solubilization and subsequent dilutions. An amount equivalent to $10 \mathrm{mg}$ of imipenem standard was accurately weighed and transferred to $100 \mathrm{~mL}$ volumetric flask. Distilled water was added to make up to volume and give the final concentration of the stock solution. This stock solution (imipenem $100 \mu \mathrm{g} / \mathrm{mL}$ ) was used during the experiment. For sample solution, the same protocol was applied starting from an amount equivalent to $10 \mathrm{mg}$ of imipenem powder for injection. Both stock solutions (100 $\mu \mathrm{g} / \mathrm{mL}$ ) were used in the subsequent dilutions.

\section{Cylinder-plate assay}

The $3 \times 3$ design was performed with 8 plates for assay, following the procedure recommended by official reference (17). The $100 \times 20 \mathrm{~mm}$ petri dishes were filled with number 11 agar in two separate layers: the base layer and the surface layer. The base layer was composed by $20 \mathrm{~mL}$ of number 11 agar and the surface layer was composed by $6 \mathrm{~mL}$ of inoculum (2\%). Six $8 \mathrm{~mm}$ cylinders were distributed over the surface layer in each plate, for subsequent application of the drug solutions.

These cylinders were filled with $200 \mu \mathrm{L}$ of standard solution or sample solution in three different concentrations of each, and then, the plates were incubated in dry air oven at $37^{\circ} \mathrm{C}$ for 18 hours. Inhibition halos were measured using a digital caliper Starret ${ }^{\circledR}$ and the data collected were analyzed for antimicrobial potency calculations, by correlating diameter of inhibition halos and log of drug concentration.

\section{Preliminary assays}

Two concentrations were tested for inoculum suspension $(1 \%$ and $2 \%$ ) in a preliminary assay with 20 plates, being 10 plates with each concentration of inoculum. At the same preliminary experiment, two ranges of concentrations for standard and sample solutions were tested: $1.0 ; 2.0$ and 4.0 $\mu \mathrm{g} / \mathrm{mL}$ and $2.0 ; 4.0$ and $8.0 \mu \mathrm{g} / \mathrm{mL}$, which was prepared from the stock solutions. In the plates with $1 \%$ of inoculum concentration, the two ranges of dilutions for standard and sample solutions were tested ( 5 plates for each group). The same procedure was done for the plates with $2 \%$ inoculum concentration. The results were compared to evaluate the microorganism growth and inhibition halo measurements.

Another preliminary experiment was performed from observation of previous results, using $2 \%$ of inoculum concentration and testing three new concentrations for standard and sample solutions: $0.5 ; 1.0$ and $2.0 \mu \mathrm{g} / \mathrm{mL}$. Here, the focus was the inhibition halo sizes, which must to be adequate for practical measurement and to avoid possible interferences.

\section{Method validation}

\section{Linearity and Precision (repeatability and intermediate precision)}

To evaluate linearity, three concentrations were prepared from the stock solutions of imipenem standard. The stock 
Drug Anal Res, 2018; 02, 08-13

solution was diluted in volumetric flasks $(10 \mathrm{~mL})$ with sterile distilled water and the final concentrations obtained was $0.5 ; 1$ and $2 \mu \mathrm{g} / \mathrm{mL}$. Linearity was assessed by constructing nine standard curves in three days of assay, being three curves a day, and each curve considered the results from eight plates. The analysis of linear regression was performing using least squares regression method, and statistical analyses to validate the results were performed using ANOVA. The repeatability (intra-day) was evaluated comparing results of three eight-plate assays, performed in the same day at the same conditions, and the intermediate precision was evaluated comparing results of three different days of experiment, being three assays of eight plates a day. Relative standard deviation (RSD) was calculated.

\section{Accuracy}

The accuracy was assayed by recovery method. Known quantities of standard solutions were added to sample solutions with nominal concentrations of $0.5 ; 1$ e $2 \mu \mathrm{g} / \mathrm{mL}$. The amount of standard added corresponds to $10 \%$ of these solutions concentrations. Then, the recovery percentages of the standard added were calculated. The results from three eight-plate assays performed in the same day were used in the calculations. Relative standard deviation (RSD) from the analyses was also calculated.

\section{Results and discussion}

\section{Methodology optimization}

The success in a method validation is widely dependent of well-established analytical conditions. In special case of microbiological methodologies, where there is a great source of variations that may interfere on analysis, is important the optimization of the conditions in order to obtain a reliable and reproductive method. The microbiological assay using the cylinder-plate assay and $3 \times 3$ design (three concentrations of standard and sample in the same plate) has been recommended by official references (16), being used for many antibiotics. The principle of this method is the drug diffusion on an agar surface inoculated with a microorganism, producing a inhibition zone of growth that is measured (20-22) and allows to determine the drug potency and to detect small variances in biological activity. Agar diffusion methods have been the methodology chosen for water-soluble antibiotics (23).

For the present study, the culture medium was selected based on similar methodologies described for others similar carbapenems: meropenem and doripenem $(13,18)$. As test microorganism, Staphylococcus epidermidis was selected due availability as well as its adequate growth in the culture media selected and reproducibility on measurement of halos. Testing inoculum concentration, the better response was observed by using the higher concentration $(2 \%)$, whose growth was better in terms of intensity and uniformity.

From this result, another preliminary experiment tested different dilutions for standard and sample using plates with $2 \%$ of inoculum concentration. The drug concentrations tested were $1.0 ; 2.0 ; 4.0 \mu \mathrm{g} / \mathrm{mL}$ and $2.0 ; 4.0 ; 8.0 \mu \mathrm{g} / \mathrm{mL}$. It is very important for a better and more reliable measure of inhibition halos to have adequate separation between them in the surface layer. For it, it is important to find a range with a good response from geometric progression used in the calculations. The two concentration ranges tested did not provide a good response in terms of reproducibility and inhibition halo sizes, as expected. In this case, it was observed a very large halo size. Based on these first preliminary results, we tested another experimental variation focused on concentration range, testing $0.5 ; 1.0$ and $2.0 \mu \mathrm{g} /$ $\mathrm{mL}$. Now, the result was satisfactory because allowed adequate separation and reproducible measure of inhibition halos, as illustrated (Figure 2). So, one additional experiment was performed with the defined analytical conditions to test the reproducibility, showing satisfactory results.

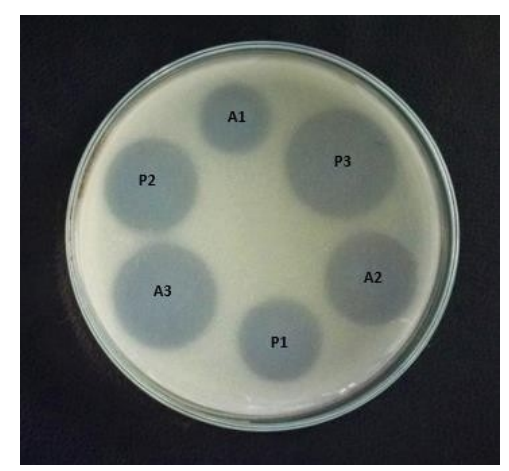

Figure 2. Illustrative image of a plate with concentration inoculum $2 \%$, containing three concentrations of imipenem standard (P) and commercial sample (powder for injection) (A): A1 and P1 for $0.5 \mu \mathrm{g} / \mathrm{mL}$; A2 and P2 for $1.0 \mu \mathrm{g} / \mathrm{mL}$; A3 and P3 for $2.0 \mu \mathrm{g} / \mathrm{mL}$. Growth on antibiotic media number 11 .

\section{Method validation}

The validation parameters studied were linearity, precision (repeatability and intermediate precision) and accuracy. All analytical procedures followed the official guidelines: $\mathrm{ICH}$ (24) and the Brazilian Health Regulatory Agency (25).

For linearity, it was constructed standard curves that correlates inhibition halo sizes with drug concentration in logarithmic values. For it, it was obtained nine standard curves in three days of analysis, being three curves a day at the same conditions. The standard curves considered each one 8 plates, totalizing 24 plates at the same day. The range of drug concentration assayed was $0.5 ; 1.0$ and $2.0 \mu \mathrm{g} / \mathrm{mL}$. The means of halo diameters for each drug concentration were $15.20 \mathrm{~mm}(\mathrm{RSD}=2.87) ; 19.45 \mathrm{~mm}(\mathrm{RSD}=1.42)$ and $23.12 \mathrm{~mm}(\mathrm{RSD}=2.76)$, respectively. In agreement to official guidelines, the $r$ value (correlation coefficient) of the standard curves must be close or equal 1.0, in order to demonstrate the best linear relation between the variables. In this work, all curves obtained a $\mathrm{r}$ value $>0.99$, being considered very satisfactory. A standard curve obtained in third day of analysis with $r=0.9991$ is illustrated on Figure 3. 
Drug Anal Res, 2018; 02, 08-13

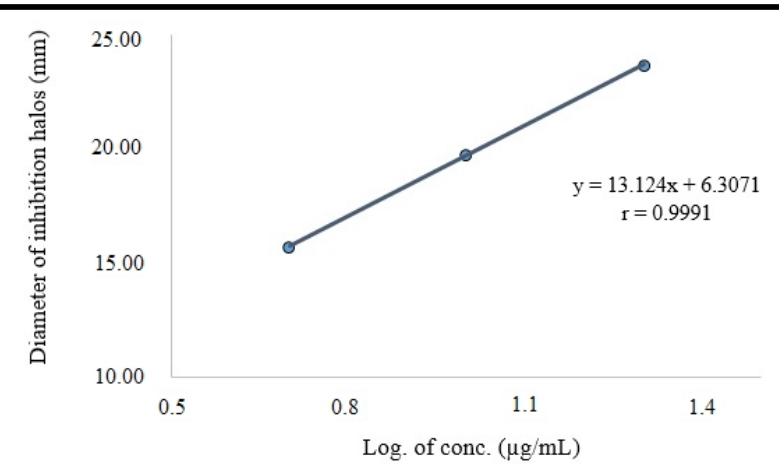

Figure 3. Standard curve correlating diameter of inhibition zone $(\mathrm{mm})$ versus logarithmic of drug concentration $(\mu \mathrm{g} / \mathrm{mL})$, obtained from microbiological assay developed to quantitation of imipenem in powder for injection.

The linear regression analysis performed by ANOVA (analysis of variance) was used to prove the linearity performance, and not shown deviations from parallelism or significant differences from the medium values ( $p$-value $>$ $0.05)$, as recommended in official guidelines $(17,19)$. The Table 1 illustrates the ANOVA applied to results of third day of analysis, considering results from 24 plates (equivalent to three assays with 8 plates).
According to official codes, the evaluation of precision can be expressed in two levels: repeatability and intermediate precision. As preconized in 3 x 3 design of cylinder plate assay, during precision both commercial sample and reference standard were analyzed in all experiments and then, the imipenem content was calculated. To access repeatability and precision intermediate, results of six assays with 8 plates were considered in three days of analysis (three assays with 8 plates a day) and the calculated RSD were satisfactory in all the cases $(\mathrm{RSD}=0.28-0.64)$. The mean drug content was $101.05 \%(\mathrm{RSD}=2.49)$ (Table 2). As described in imipenem monograph found in US pharmacopeia, the powder for injection must to contain 90$115 \%$ of imipenem and cilastatin (16).

Microbiological assay is classified as biological assay and generally, have a highly variation of results (26) in comparison with analytical methods like HPLC. Despite of this, the RSD values obtained in the purposed method were adequate, being below to $5 \%$ for all experiments, showing that this microbiological assay is suitable to determine imipenem in drug formulation despite the inherent variation of biological assays.

Table 1. Analysis of variance (ANOVA) from quantitative results of linearity (third day) obtained during validation of a microbiological assay for imipenem in powder for injection.

\begin{tabular}{|c|c|c|c|c|c|}
\hline Variation sources & $\begin{array}{l}\text { Degrees of } \\
\text { freedom }\end{array}$ & Sums of Squares & Mean Squares & F-calculated & F-value \\
\hline Preparation & 1 & 0.005 & 0.005 & 3.88 & 4.12 \\
\hline Regression & 1 & 509.60 & 509.60 & 395424.10 & 4.12 \\
\hline $\begin{array}{l}\text { Desviation from } \\
\text { paralelism }\end{array}$ & 1 & 0.000 & 0.000 & 0.010 & 4.12 \\
\hline Quadratic & 1 & 0.003 & 0.003 & 2.53 & 4.12 \\
\hline Quadratic difference & 1 & 0.000 & 0.000 & 0.013 & 4.12 \\
\hline $\begin{array}{l}\text { Difference between } \\
\text { doses }\end{array}$ & 5 & 509.61 & 101.92 & 79086.11 & 2.48 \\
\hline $\begin{array}{l}\text { Difference between } \\
\text { plates }\end{array}$ & 7 & 0.009 & 0.001 & 0.951 & 2.29 \\
\hline
\end{tabular}

Table 2. Results obtained from intermediate precision (inter-day) and repeatability (intra-day), worked during validation of a microbiological assay for quantitation of imipenem in drug formulation. Data expressed as potency (\%) and RSD.

\begin{tabular}{|c|c|c|c|}
\hline & \multicolumn{3}{|c|}{ Repeatability } \\
\hline & \multicolumn{3}{|c|}{ Antimicrobial Potency (\%) ${ }^{\text {a }}$ Mean Potency (\%) RSD (\%) } \\
\hline & 104.16 & & \\
\hline \multirow[t]{2}{*}{ Day 1} & 104.16 & 104.33 & 0.28 \\
\hline & 104.67 & & \\
\hline & 98.59 & & \\
\hline \multirow[t]{2}{*}{ Day 2} & 99.81 & 99.45 & 0.76 \\
\hline & 99.96 & & \\
\hline \multirow{4}{*}{ Day 3} & 98.98 & & \\
\hline & 98.99 & 99.35 & 0.64 \\
\hline & 100.09 & & \\
\hline & Intermediate precision & 101.05 & 2.49 \\
\hline
\end{tabular}

${ }^{a}$ Each result is a mean from three assays of eight plates.
For accuracy, recovery testing was performed according to described for other microbiological methods applied to carbapenems $(13,19)$. Here, the recovery percentage was between $101.70-105.20 \%$ (mean recovery $=104.86 \%$ ) with $\mathrm{RSD}=2.95$ (Table 3), each result considering three assays with six plates each one. So, the recovery percentage obtained was considered satisfactory and the method demonstrated to be accurate for quantitative estimation of imipenem. 
Drug Anal Res, 2018; 02, 08-13

Table 3. Mean values (\%) of recovery test obtained from microbiological assay applied to quantitation of imipenem in powder for injection.

\begin{tabular}{cccc}
\hline $\begin{array}{c}\text { Sample } \\
\text { concentration } \\
(\boldsymbol{\mu} \mathbf{g} / \mathbf{m L})\end{array}$ & $\begin{array}{c}\text { Standard added } \\
(\boldsymbol{\mu g} / \mathbf{m L})\end{array}$ & $\begin{array}{c}\text { Recovered } \\
\text { concentration } \\
(\boldsymbol{\mu g} / \mathbf{m L})\end{array}$ & $\begin{array}{c}\text { Mean of recovery } \\
(\%)\end{array}$ \\
\hline 0.5 & 0.05 & 0.051 & 101.70 \\
\hline 1.0 & 0.10 & 0.107 & 107.90 \\
\hline 2.0 & 0.20 & 0.210 & 105.20 \\
\hline & RSD (\%) & & 2.95 \\
\hline
\end{tabular}

${ }^{a}$ Each result is a mean from three assays of six plates.

\section{Conclusions}

The commercial samples of imipenem were quantified by the purposed microbiological assay, whose validation was considered satisfactory. Linearity, precision and accuracy were the parameters studied and the results were satisfactory in terms of official limits. So, the cylinder-plate assay method can be an analytical option for imipenem quantitation, in special when the biological potency is useful for a better comprehension about drug quality. This bioassay can be also performed in a complementary view about physicochemical methods like UV spectrophotometry or HPLC.

\section{Conflict of Interest}

The authors declare the absent of any conflict of interest in all the results presented in this paper.

\section{Acknowledgements}

The authors thank to CAPES for financial support and to Universidade Federal do Rio Grande do Sul (UFRGS) for all structure provided.

\section{References}

1. Nicolau DP. Carbapenems: a potent class of antibiotics. Expert Opin Pharmacother. 2008; 9: 23-27.

2. Zhanel GG, Simor AE, Vercaigne L, Mandell L. Imipenem and Meropenem: Comparison of in vitro activity, pharmacokinetics, clinical trials and adverse effects. Can J Infect Dis. 1998; 9: 215-228.

3. Rodloff AC, Goldstein EJC, Torres A. Two decades of Imipenem therapy. J Antimicrob Chemother. 2006; 58: 916-929.

4. Hellinger WC, Brewer NS. Carbapenems and Monobactams: Imipenem, Meropenem and Aztreonam. Mayo Clin Proc. 1999; 74: 420-435.
5. McGregor JA, Christensen FB, French JI. Intramuscular Imipenem/Cilastatin treatment of upper reproductive tract infection in women: efficacy and use characteristics. Chemotherapy. 1991; 37: 31-36.

6. Legrand T, Chhun S, Rey E, Blanchet B, Zahar JR, Lanternier F, et al. Simultaneous determination of three carbapenems antibiotics in plasma by HPLC with ultraviolet detection. J Chromatogr B. 2008; 875: 551556.

7. Dailly E, Bouquié R, Deslandes G, Jolliet P, Floch RL. A liquid chromatography assay for a quantification of doripenem, ertapenem, imipenem e meropenem concentrations in human plasma: Application to a clinical pharmacokinect study. J Chromatogr B. 2011; 879:

1137-1142.

8. Torres-Fernández R, Navarro MV, López MAB, Mochón MC, Sánchez JCJ. Urea as new stabilizing agent for imipenem determination Electrochemical study and determination of imipenem and its primary metabolite in human urine. Talanta. 2008; 77: 241-248.

9. El-Kosasy AM, Abdel-Aziz O, Magdy N, El Zahar NM. Spectrophotometric and chemometric methods for determination of imipenem, ciprofloxacin hydrochloride, dexamethasone sodium phosphate, paracetamol and cilastatin sodium in human urine. Spectrochim Acta A Mol Biomol Spectrosc. 2016; 157: 26-33.

10. Parra A, Garcia-Villanova J, Ródenas V, Gómez MD. First- and second-derivate spectrophometric determination of imipenem and cilastatin in injections. $\mathrm{J}$ Pharm Biomed Anal. 1993; 11: 477-482.

11. Tabbouche OS, Soukkariyyeh I. First-order derivate ultraviolet spectrophotometry of imipenem-cilastatin formulations. J Taibah Univ Sci. 2014; 9: 178-181.

12. Vikram P, Shanmugasundaram P. A highly sensitive stability-indicating UPLC method for the determination of related substamces in imipenem and cilastatin for injection. Int J Pharma Bio Sci. 2016; 7: 144-154.

13. Mendez ASL, Weisheimer V, Oppe TP, Steppe M, Schapoval EES. Microbiological assay for the determination of meropenem in pharmaceutical dosage form. J Pharm Biomed Anal. 2005; 37: 649-653.

14. Mahmoudi A, Fourar REA, Boukhechem MS, Zarkout S. Microbiological assay for the analysis of certain macrolides in pharmaceutical dosage forms. Int $\mathrm{J}$ Pharm 2015; 491: 285-291.

15. Staub I, Schapoval EES, Bergold AM. Microbiological assay of ketoconazole in shampoo. Int J Pharm. 2005; 292: 195-199. 
16. The United States Pharmacopoeia, 32st ed.; United States Pharmacopoeial Convention: Rockville, 2009.

17. Brazilian Pharmacopeia, 5th ed.; Anvisa: Brasília, 2010.

18. European Pharmacopoeia, 8th ed.; Council of Europe: Strasbourg,

2014.

19. Führ F, Paliosa PK, Costa MCN, Garcia CV, Schapoval EES, Steppe M, et al. Bioassay applied to quantitative determination of Doripenem in powder for injectiondegradation kinetics and comparison to HPLC and CE methods. Curr Pharm Anal. 2013; 9: 244-251.

20. Gavin JJ. Analytical microbiology: II The diffusion methods. Microbiological process reports. Appl Microbiol. $\quad$ 1956; $\quad$ : 25-33.

21. Shah VP, Midha KK, Dighe S, McGilveray IJ, Skelly JP, Yakobi A, et al. Analytical methods validation: Bioavailability, bioequivalence and pharmacokinetic studies. J Pharm Sci. 1992; 81: 309-312.
22. Costa MCN, Barden AT, Andrade JMM, Oppe TP, Schapoval EES. Quantitative evaluation of besifloxacin ophthalmic suspension by HPLC, application to bioassay method and cytotoxicity studies. Talanta. 2014; 119: 367-374.

23. Christ AP, Machado MS, Ribas KG, Schwarzbold AV, Silva CB, Adams AIH. A fully validated microbiological assay for daptomycin injection and comparison to HPLC method. Braz J Pharm Sci. 2015; 511: 775-783.

24. ICH. In: Validation of Analytical Procedures, Text and Methodology - Q2 (R1), International Conference on Harmonization of Technical Requirements for the Registration of Pharmaceutical for Human Use, 2005.

25. Brazilian Health Regulatory Agency (ANVISA). Resolution: RE $n^{\circ} 166$, July $24^{\text {th }}, 2017$.

26. Breier AR, Garcia CV, Oppe TP, Steppe M, Schapoval EES. Microbiological assay for azithromycin in pharmaceutical formulations. J Pharm Biomed. Anal. 2002; 29: 957- 961 . 